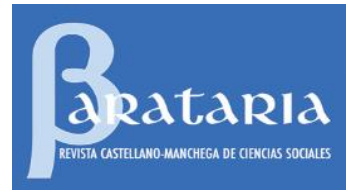

\title{
LA PROTECCIÓN AL WHISTLEBLOWER: RETOS PARA LA IMPLEMENTACIÓN EN ESPAÑA
}

\section{WHISTLEBLOWER PROTECTION: CHALLENGES FOR IMPLEMENTATION IN SPAIN}

\author{
Manuel Villoria \\ Universidad Rey Juan Carlos \\ manuel.villoria@urjc.es \\ Orcid: 0000-0002-1055-1995
}

Recibido/Received: 14/03/2021

Modificado/Modified: 21/04/2021

Aceptado/Accepted: 23/9/2021

\section{RESUMEN}

La trasposición de la Directiva europea 2019/1937, sobre protección a las personas que denuncian incumplimientos del derecho de la Unión, aunque recoge en su articulado las mejores prácticas internacionales, supone un reto de implementación enorme.

El artículo trata de explicar las más importantes demandas organizativas, financieras y de gobernanza que genera su implantación. Con ello, se recupera el foco en la implementación de políticas como un área que merece la atención de las policy sciences. En este estudio de caso, basado en experiencia empírica internacional y en encuestas propias, se demuestra que, a pesar de las normas, para alcanzar un mínimo éxito en la protección al alertador, es preciso considerar variables como la complejidad del tema, la multiplicidad de actores, la amplitud de las posibles demandas, la dualidad de sistemas internos y externos, públicos y privados, y la heterogeneidad de espacios de protección y sanción.

Sumario: I. Introducción. II. El reto de la implementación. III. Los recursos y la gobernanza del sistema. 3.1 ¿Qué denuncias generan protección? 3.2 La gobernanza del sistema. IV. Teoría causal y reglas de decisión. El problema de la gestión interna de las denuncias. ¿Por qué la gestión interna es a menudo insuficiente? V. Variables no normativas. VI. Conclusiones

\section{PALABRAS CLAVE:}

Denunciante. Implementación. Gobernanza. Denuncias de incumplimientos. Directiva europea 2019/1937.

\begin{abstract}
The transposition of the European Directive 2019/1937 on protecting persons who report EU law breaches, although it includes international best practices in its norms, represents an enormous implementation challenge. This article tries to explain the most critical organizational, financial, and governance demands generated by its implementation. In doing so, the focus on policy implementation is regained as an area that deserves the attention of policy sciences. In this case study, based on international empirical experience and own surveys, it is shown that, despite the rules, in order to achieve a minimum success in more whistleblower protection, it is necessary to consider variables such as the complexity of the issue, the multiplicity of actors, the breadth of
\end{abstract}


possible demands, the duality of internal and external systems, public and private, and the heterogeneity of protection and sanction spaces.

\section{KEYWORDS:}

Whistleblower. Implementation. Governance. Reporting of breaches. Directive (EU) 2019/1937.

\section{CONTENTS:}

I. Introduction. II. The challenge of implementation. III. Resources and governance of the system. 3.1 Which complaints generate protection? 3.2 The governance of the system. IV. Causal theory and decision rules. The problem of internal complaint management: Why is internal management often insufficient? V. Non-normative variables. VI. Conclusions

\section{INTRODUCCIÓN}

La denuncia de actos corruptos e inmorales desde dentro de las organizaciones es una práctica que tiene miles de años. La protección de los que denuncian, por desgracia, es mucho más reciente. La primera norma es de 1778. Sólo siete meses después de la firma de la Declaración de Independencia, el Congreso Continental aprobó lo que Allison Stanger ha llamado la "primera ley de protección de denunciantes del mundo" (2019a:1). A partir de ahí, siguiendo de nuevo a Stanger (2019a), las normas generadas en EE.UU para proteger a los denunciantes son muy numerosas: La False Claim Act de 1863, la Ley de Reforma de la Función Pública de 1978, la Ley del Inspector General de 1978, o la Ley de Protección de Denunciantes de 1989. Finalmente, como consecuencia de los escándalos financieros en grandes empresas (ENRON, Tyco, etc.) en 2002 se aprobó La Ley Sarbanes-Oxley, también conocida como la Ley de Reforma de la Contabilidad Pública de Empresas y de Protección al Inversionista, la cual establece toda una serie de normas para proteger a los whistleblower en el sector privado.

No obstante, a pesar de toda esta abundante legislación, el análisis de casos específicos de whistleblowing aporta una imagen poco optimista sobre el nivel real de protección en EE.UU. (ver, Glazer y Glazer, 2001; Alford, 2002; Dworkin, 2007; Stanger, 2019b). Mientras, la expansión de normas para proteger a denunciantes no ha hecho sino incrementarse. Actualmente (2021), hay 62 países con normas de protección de denunciantes. Dentro de la OCDE (2016), no hay disposiciones legales que protejan específicamente a los denunciantes del sector público en tan sólo el 15,6\% de los países. En concreto en: Finlandia, Polonia, España, Suecia y República Checa. Existe una Ley dedicada a la protección de los denunciantes en el sector público en el 40,6\% de los países. Finalmente, existen diversos grados de protección de los denunciantes, en una o más leyes, relacionadas específicamente con la protección de las denuncias o la prevención de las represalias contra los denunciantes en el $43.8 \%$ restante. A pesar de esta expansión, el Grupo de Trabajo de la OCDE sobre el Soborno en transacciones internacionales descubrió, en una encuesta de 2014, que 27 de los 41 países signatarios de la Convención contra el Soborno carecían de leyes, o las que tenían era ineficaces, para proteger a los alertadores del sector privado que denunciaban sospechas de soborno en los negocios internacionales.

En su reciente informe sobre las leyes de 37 países, Feinstein y Devine (2021) nos muestran que, en general, la mayoría de los denunciantes no tienen éxito formalmente en las denuncias por represalias. En los países en los que se presentan casos con cierta 
frecuencia (30 o más casos), algo menos del 13\% de los denunciantes obtuvieron decisiones formales y definitivas favorables. En los 37 países combinados, la tasa de éxito global fue del 21\% (80 victorias de 379 decisiones sobre el fondo). Incluso cuando los denunciantes se imponen oficialmente, a menudo "pierden ganando" debido a las escasas indemnizaciones económicas, los elevados costes y los largos procedimientos para resolver los casos de represalias. No obstante, en términos más positivos, los datos de victorias y derrotas infravaloran la eficacia de las leyes sobre denunciantes, porque una parte significativa de los casos se resuelven mediante acuerdos que no se producirían en ausencia de la protección legal.

Dicho esto, la pregunta es ¿qué se puede hacer para mejorar el sistema? El informe citado de Feinstein y Devine establecen 20 buenas prácticas para mejorar las normas existentes. La Directiva europea (UE) 2019/1937 (en adelante la Directiva) prácticamente asume todas ellas, lo que nos aporta dosis de optimismo de cara a su trasposición a la normativa española. La recomendación principal del informe es redactar leyes que reflejen las mejores prácticas mundiales y aplicarlas de buena fe. Sin embargo, el informe también señala que las leyes con mejores prácticas serán ineficaces sin el apoyo o la supervisión pública de estos derechos, y eso no es posible sin transparencia, educación y evaluación de la implementación e impacto. Como vemos, al final, el informe nos abre el debate de la calidad de la implementación. A partir de aquí, el texto se centrará en analizar el reto de la implementación de la directiva en el caso español, con especial énfasis en el estudio de sus problemas de gobernanza y las limitaciones de las investigaciones internas, generando algunas propuestas y concluyendo con la presentación de algunos debates que se abren. Este estudio de caso se funda, en gran medida, en investigaciones empíricas propias sobre corrupción en el sector privado (Palomo et al. 2018).

\subsection{EL RETO DE LA IMPLEMENTACIÓN}

Más allá de los debates sobre si se puede dividir una política en fases bien delimitadas, lo que está claro, a partir de numerosa investigación empírica, es que las leyes no agotan el contenido de una "política", igual que la implementación no es lo único importante de la policy. Previamente a llevar adelante la política es preciso que exista el acto cognitivo de definir qué se necesita hacer para resolver el problema identificado y que se tome la decisión de asumir una específica solución, sea de forma racional (Dror, 1983) o incremental (Lindblom, 1965). La buena implementación se genera ya en la fase de diseño, creando las condiciones para que sea posible (por ejemplo, reservando presupuesto adecuado para la ejecución o estableciendo una evaluación obligatoria de la implementación de la norma). Pero, en todo caso, esta fase es extraordinariamente compleja y en ella se estrellan muchos de los sueños de cambio institucional. La implementación puede cambiar completamente el objetivo de una política, sus prioridades y sus pretendidas consecuencias. En ella caben reglamentos, órdenes, decisiones administrativas, sentencias judiciales, el cumplimiento, incumplimiento o cumplimiento parcial por los destinatarios, los impactos percibidos y las revisiones de lo que se va haciendo en función de consecuencias imprevistas, efectos indeseados o de los análisis evaluativos que van proporcionando datos sobre el "implementation gap" (Hill y Hupe, 2002). Considerando sólo el enfoque top-down en la implementación (existe un enfoque bottom-up que insiste en que los agentes públicos operativos pueden en el día a día de la 
implementación transformar los diseños hechos arriba), los requisitos para una adecuada implantación son muy numerosos y de difícil control (ver figura 1).

Figura 1. Diagrama de Flujo de las variables del proceso de Implementación

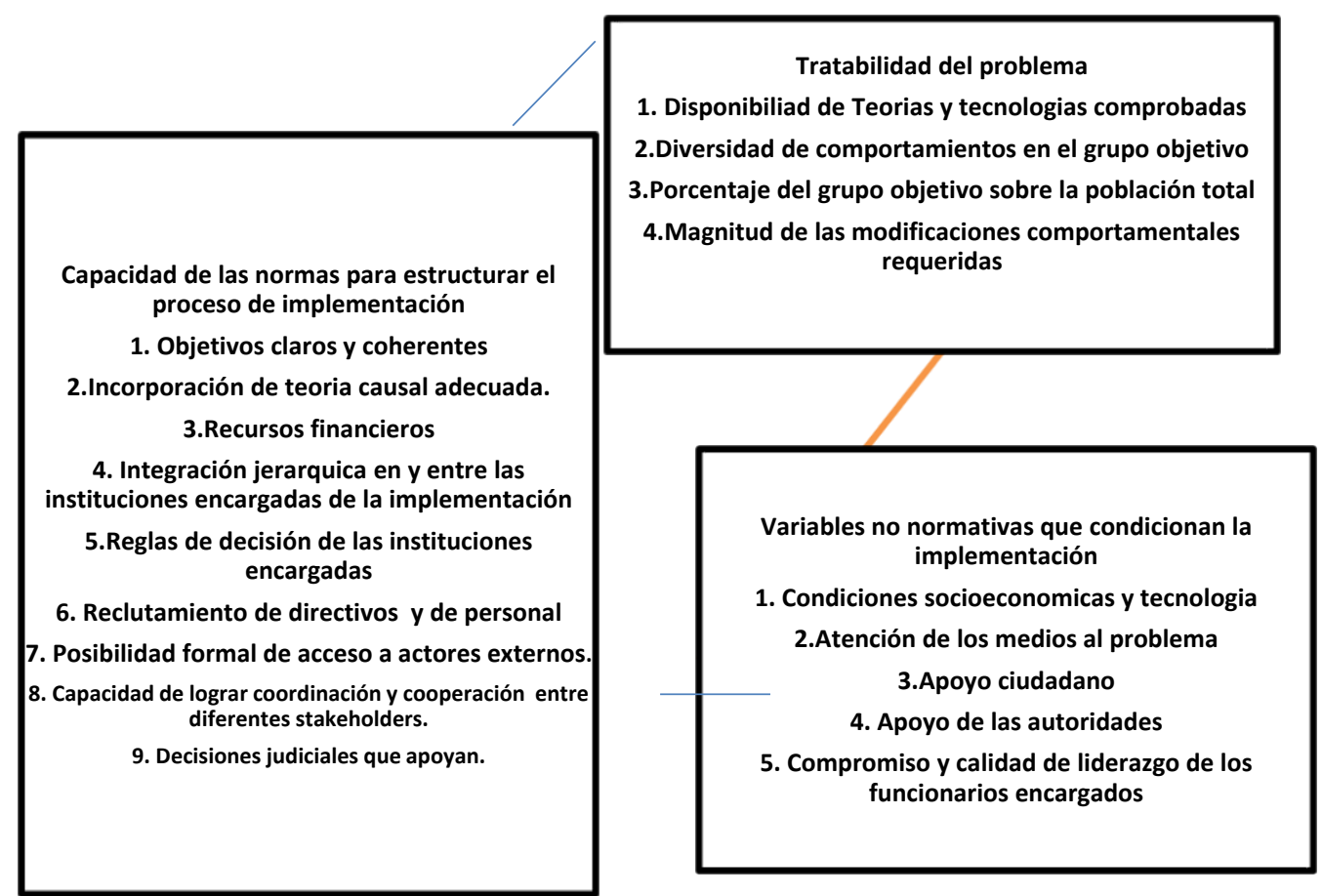

Fuente: Sabatier \& Mazmanian, 1980, y elaboración propia.

Cuando estamos hablando de una política como la de proteger denunciantes y alertadores, siguiendo el marco de la directiva europea, estamos hablando de una política de una complejidad inmensa, pues implica, para que cumpla sus objetivos, concertar actores y recursos de diferentes niveles de gobierno, del sector público, privado y nogubernamental y en todo el territorio del Estado. Es evidente, como ya nos indicaron Pressman y Wildavsky, que cuanto más actores existan y más alejados del centro de decisión estén, más posibles puntos de veto y espacios de negociación existirán y más difícil será que la política alcance plenamente los objetivos para los que nació (1984). En esta política (o mejor, en este componente del subsistema de políticas de buena gobernanza) la tratabilidad del problema es baja. Afecta a miles de organizaciones, a millones de personas insertas en tipos de organizaciones muy diversos y conlleva un cambio organizativo y cognitivo bastante profundo. De acuerdo con los datos de nuestras encuestas (Palomo et al., 2018), apenas el 14\% de las empresas españolas forman en políticas anti-corrupción o similares, y no llega a un $25 \%$ las que dicen que tienen sistemas para proteger a denunciantes (sea el que sea este sistema). En el sector público no existen apenas sistemas de gestión de la integridad que incorporen espacios de denuncia, 
protección a alertadores y denunciantes, formación en ética, encuestas de clima ético, etc. En gran medida, se trata de incorporar al ordenamiento español un instrumento de otra realidad jurídica (Pérez Monguió, 2019: 353). En suma, estamos ante un cambio muy profundo que se topará con caminos de dependencia e inercias bastante fuertes. De ahí la necesidad de ir evaluando la implementación de la norma de forma anual.

\section{LOS RECURSOS Y LA GOBERNANZA DEL SISTEMA}

\section{1 ¿QUÉ DENUNCIAS GENERAN PROTECCIÓN?}

Analizando las variables que hemos sistematizado en la figura 1, un primer elemento de debate es el de qué tipo de denuncias se admitirán como investigables y generadoras de protección. En general, las denuncias de delitos generan protección, tanto en el sector público como en el privado. Pero, después, hay un inmenso campo de posibilidades. La Directiva habla de "denuncias en fraudes e incumplimientos normativos" en una serie de servicios e industrias. De acuerdo con la normativa comparada, las denuncias por despilfarro en el sector público también deberían ser incluidas pues, aunque no son fraude ni corrupción strictu sensu, son acciones que dañan gravemente el interés general. Y junto a ello, las denuncias por incumplimiento de los códigos éticos también deben incluirse, pues los códigos son elementos esenciales de los sistemas de compliance y de integridad y marcan el deber ser del comportamiento en la organización. Vinculado a ello, las denuncias por incumplimiento de los deberes de buena administración en el sector público no pueden quedar al margen de la investigación y protección al denunciante. Existe un derecho subjetivo a una buena administración que puede hallarse explícito en el "artículo 41 de la Carta de Derechos Fundamentales de la Unión Europea y en diversa legislación española (Ponce, 2017b: 232).

Con esta amplitud de posibles denuncias, las demandas de recursos financieros y humanos para la gestión de las investigaciones y de las posibles protecciones son enormes. Parece claro que, sin recursos financieros suficientes, esta norma será un fraude. Si no se puede proteger jurídicamente a los alertadores, si no se les puede indemnizar, si no hay personal para apoyarlos psicológicamente es evidente que la norma no cumplirá sus objetivos. Pero este tema de los recursos financieros está íntimamente unido al problema de los recursos humanos. La norma exigirá la creación de gestores de integridad en las organizaciones públicas y compliance officers en las privadas, además de nuevos órganos de control. En lo público, esto implica un reforzamiento de las unidades de inspección enorme, de forma que puedan investigar adecuadamente las denuncias; un reforzamiento no sólo en número, sino también en competencias y protección frente a represalias. En el ámbito local, como luego veremos, se tendrá que repensar el sistema de control de la integridad de forma casi completa. Otro aspecto es el del papel del poder judicial en esta política; será preciso un amplio programa de formación y sensibilización para que sus decisiones no entorpezcan la aplicación y generen la sensación de que los denunciantes no están protegidos.

\subsection{LA GOBERNANZA DEL SISTEMA}

Veamos ahora la complejidad de la gobernanza del sistema. Para ello, comprobemos mediante la figura 2 los múltiples actores y niveles, los problemas jerárquicos y de 
coordinación y cooperación que se vislumbran. Dentro de lo público, habría que distinguir denuncias internas que implican incumplimiento de normas que pueden conllevar delitos vinculados a la corrupción, denuncias por incumplimiento de normas que pueden implicar responsabilidades disciplinarias (normalmente, en estos casos, denuncias entre compañeros), denuncias por incumplimiento de otras normas, o por incumplimiento de principios de buena administración (por ejemplo, el de due diligence), etc... Internamente, alguien se tendrá que encargar de diferenciar unas y otras y remitirlas para investigación a órganos adecuados o bien investigarlas directamente. Este órgano interno habrá que definirlo claramente y, en su caso, otorgarle capacidad de investigación independiente de los hechos.

¿Existen esos órganos internos? Todavía no. Se necesita crear un tipo de puesto que sea capaz de analizar todas las posibles denuncias y definir el camino idóneo de investigación para cada una de ellas, un puesto con la responsabilidad de gestionar el buzón interno de denuncias y garantizar la anonimidad o la confidencialidad, según los casos. Normalmente, este mismo órgano debería ser capaz de realizar una primera investigación preliminar y, a partir de ahí, diferenciar, en su caso, los órganos externos o internos de investigación requeridos, que pueden ser desde la Fiscalía a las Agencias antifraude, pasando por el Tribunal de Cuentas (el juicio por alcance) o el instructor de expediente disciplinario, entre otros, además de solicitar la suspensión cautelar del procedimiento administrativo correspondiente si hay ilicitudes (Vestri, 2019). Generar todo este tipo de puestos en miles de organizaciones públicas, formarlos y, desde luego, aportarles protección para que no se conviertan ellos mismos en represaliados será una labor hercúlea. Probablemente, la implementación de la ley abrirá el paso a futuras reformas institucionales que, si se adoptan, mejorarán enormemente la legalidad, imparcialidad, eficacia y eficiencia de nuestras administraciones. 
Figura 2. La gobernanza del sistema

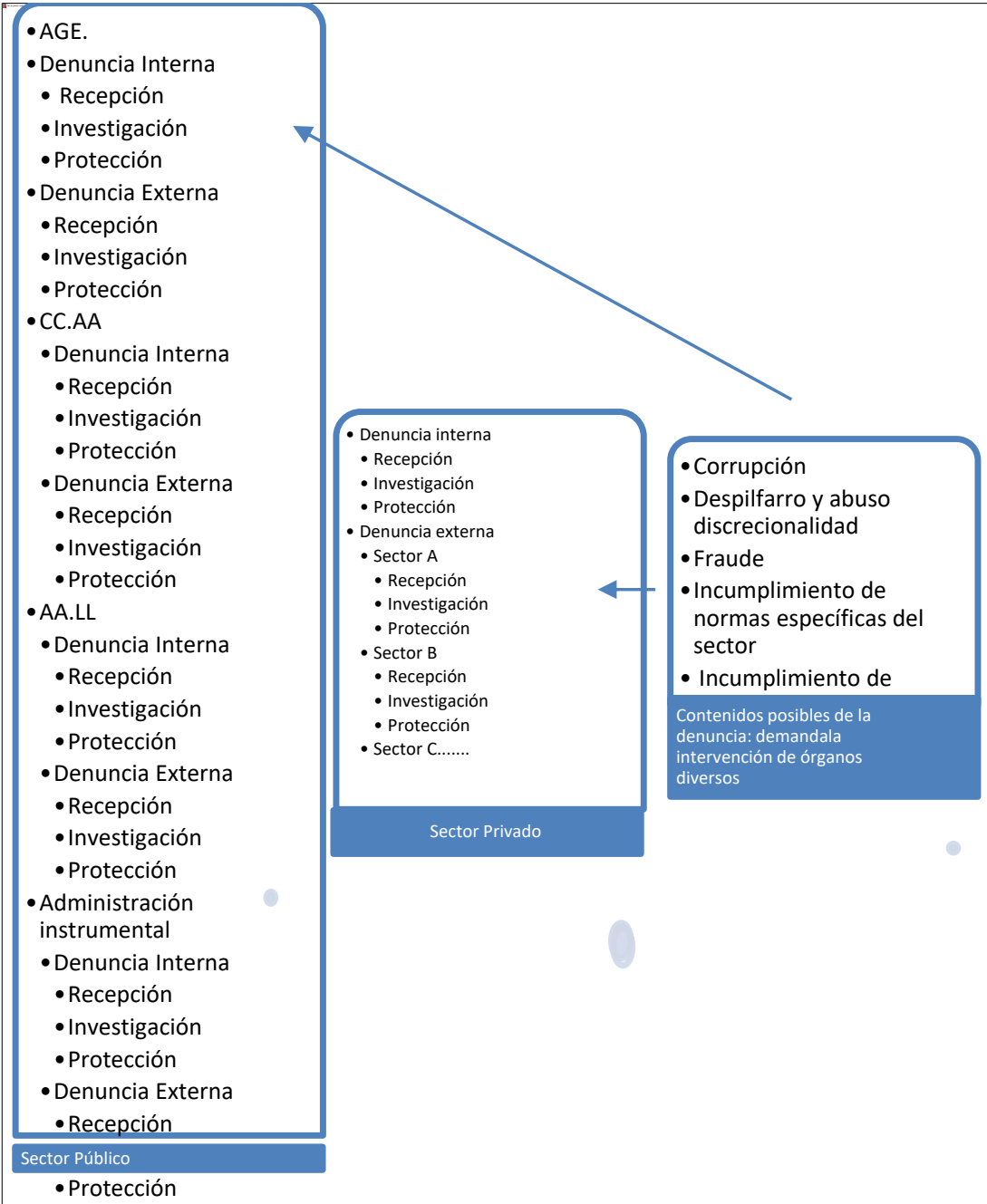

Fuente: elaboración propia

En cuanto a la denuncia externa, parece claro que, si se trata de casos de corrupción, exista un órgano especializado encargado de su investigación previa a nivel nacional y, en su caso, autonómico (Benítez Palma, 2018). Pero no está nada claro que una Agencia anticorrupción pueda ni deba investigar todas las denuncias por incumplimientos vinculados a problemas disciplinarios, o por incumplimiento de los principios de buena administración, de las normas de seguridad e higiene o medioambientales, etc. Sobre las denuncias por despilfarro no trivial, si se incluyeran en la norma, habría que definir muy bien qué se considera despilfarro no trivial y qué consecuencias tienen dichas conductas. Ahora 
mismo, es un tipo de conducta que ni está claramente tipificada en la legislación penal, ni en la contable, ni siquiera en la normativa sobre responsabilidad patrimonial, más bien queda dentro del ámbito de la discrecionalidad política, lo que produce total impunidad. En España se han despilfarrado en infraestructuras, desde 1995 a 2018, de acuerdo al estudio de Romero et al., "más de 80.000 millones de euros (las obligaciones contraídas superan los 97 000), que se han gastado mal o se han comprometido en infraestructuras ociosas, infrautilizadas, innecesarias o cerradas y en proyectos o eventos efímeros o inútiles" (2018: 35). Parece lógico que se trate de evitar la continuidad de estas ineficiencias mediante la denuncia de actividades despilfarradoras, la investigación de las mismas y la sanción correspondiente. A quién le corresponda tal labor es complejo de definir, pues el Tribunal de Cuentas, la Oficina Independiente de Regulación y Supervisión de la Contratación, la CNMC pueden todos decir algo. Nuevamente, es preciso clarificar este importantísimo tema. En todo caso, cualquier Agencia que investigue tiene que tener capacidades e independencia para poder hacer su trabajo y debe evitarse la descoordinación y confusión de permitir que más de una agencia reciba denuncias y abra investigaciones por corrupción, fraude o despilfarro (Ponce, 2017a).

Dentro de lo privado, las denuncias internas por actividades de corrupción entre privados o con el sector público han de tener un camino semejante a las denuncias de corrupción en lo público, o bien pasan directamente a la fiscalía, o se remiten desde la empresa a la fiscalía, o pasan a un órgano público de investigación preliminar (Agencias anti-corrupción). No obstante, es normal que estas denuncias (sobre todo las de corrupción entre privados), se sustancien internamente con despidos o ceses y no se dé información al exterior. El papel de los compliance officers aquí es esencial, pero cuando estas personas tienen que denunciar al administrador principal o al consejo su nivel de protección deja mucho que desear. En todo caso, la regulación sobre esta figura en España es muy reducida y se deduce del Código penal, así el artículo 31 bis, número 2, condición $2^{\mathrm{a}} \mathrm{CP}$, señala que su función es la supervisión del funcionamiento y del cumplimiento del modelo de prevención implantado, debiendo dar información al organismo encargado de vigilar el funcionamiento y observancia del modelo de prevención. Debería regularse mejor la figura, garantizar mejor su protección y definirse claramente su responsabilidad. No hay que olvidar que este tipo de puesto también se debe incorporar al sector público empresarial.

En cuanto a las denuncias externas, la diversidad enorme de actividades industriales, comerciales y de servicios hace que las denuncias externas puedan tener caminos muy diferentes de investigación. No es lo mismo una denuncia por actividades de colusión, que deberá pasar a la CNMC, que una denuncia por incumplimiento de normas en el ámbito de las centrales nucleares, que debería pasar al Consejo de Seguridad Nuclear, por ejemplo. La propia Directiva europea ya nos advierte que la Oficina Europea de Lucha contra el Fraude (OLAF), la Agencia Europea de Seguridad Marítima (AESM), la Agencia Europea de Seguridad Aérea (AESA), la Autoridad Europea de Valores y Mercados (AEVM) y la Agencia Europea de Medicamentos (EMA) disponen de canales y procedimientos de denuncia externa. En suma, que hablar de forma generalizada de denuncias es una forma de simplificación que requiere, posteriormente, un detalle mucho mayor: qué tipo de denuncia, en qué sector, a quién acudir externamente, qué tipo de investigación realiza cada organismo externo, qué consecuencias hay por los incumplimientos. Y más aún, una parte importante de las empresas (las de menos de 250 trabajadores) no tendrán, de 
acuerdo a la Directiva, que introducir sistemas de denuncia propios, por lo que dependerán plenamente de las investigaciones externas. Finalmente, habrá denuncias internas que no deban salir del ámbito organizativo por no afectar de forma mínimamente relevante a los intereses generales, por ejemplo, las denuncias entre compañeros por incumplimientos de códigos éticos sin consecuencias hacia clientes o sociedad (Brown, et al., 2008).

Del mismo modo, la protección al alertador o denunciante puede tener órganos internos y externos responsables de tal labor diferentes, no sólo entre el ámbito privado y público, sino también entre los diferentes entes públicos y privados. No hay que olvidar que en la protección al denunciante o alertador hay, al menos, tres componentes diferentes. Primero está la protección de la anonimidad o confidencialidad; segundo, está la protección strictu sensu, con la activación de los diferentes mecanismos de protección en caso de que se conozca al denunciante; tercero, está la investigación de la represalia y la sanción, en su caso, al que la comete. Estos tres componentes podrían ser responsabilidad de órganos diferentes, aunque no sea conveniente. Para empezar, el órgano interno responsable de la protección y confidencialidad en el ámbito de la AGE no será igual que en el ámbito local, pues no existe un cuerpo de inspectores internos en la Administración local que puedan llevar a cabo la labor de análisis del caso y definición de las medidas de protección. Muy importante es que este órgano, sea el que sea, ha de tener capacidad para poder sancionar a los responsables de las represalias al denunciante, o, al menos, para solicitar las sanciones. Ciertamente, este órgano interno puede tener difícil solicitar sanciones para la cúpula de un ministerio donde radica y está sometido a jerarquía. Del mismo modo, en el ámbito local, si tal labor correspondiera al secretario municipal también existiría una tensión entre el principio de jerarquía y el de protección que daría lugar a situaciones muy conflictivas. En el ámbito de la Administración instrumental, las empresas públicas no tendrían probablemente una situación distinta de las empresas privadas, debiendo contratar un compliance officer y dándole la protección y capacidad de acción que su puesto requiere, lo cual, de nuevo, es difícil de garantizar. En general, la dificultad será tanto mayor cuanto más altos estén en la jerarquía administrativa los denunciados y cuánto más grave sea la acusación.

Por todo ello, se hace difícil poder poner en manos de órganos internos la protección de los denunciantes o alertadores. Estos órganos internos se encuentran con presiones muy fuertes para que desvelen los datos que tienen sobre la identidad de los denunciantes. Si mantienen su compromiso de confidencialidad, será difícil que el denunciante sea represaliado, de ahí que sea tan importante esta labor de guardianes de la identidad de los whistleblowers. Si la identidad se conociera y el alertador fuera represaliado, estos órganos internos no pueden declarar nulo un despido, por ejemplo, menos aún sancionar a los que represalian cuando estos pertenecen a la cúpula de la organización. En consecuencia, la protección a los whistleblowers requiere de órganos externos con independencia, competencias de sanción fuertes y capacidad de aguantar presiones (para un análisis de los modelos autonómicos y los requisitos de buenas prácticas ver Capdeferro, 2020; Sierra, 2020b; Ponce, 2017 ; Garrido Juncal, 2019). Sin ellos, todo el sistema generará una falsa sensación de protección que, posteriormente, en la práctica será inexistente.

Todo esto que hemos señalado para el sector público es aún más evidente en el sector privado. Los órganos internos tienen muy difícil proteger a los denunciantes y sancionar a los que represalian cuando los hechos denunciados corresponden a lo que se denominan 
prácticas institucionalizadas de fraude o corrupción. Cuando el fraude o corrupción vienen desde arriba, en las denominadas organizaciones corruptas, la lucha contra estas patologías necesita de ayuda externa. Un órgano fundamental para la protección externa a los denunciantes será la Inspección de trabajo, como ya ocurre en Italia (ver Sierra, 2020a), la cual debe estar capacitada también para sancionar las represalias en el ámbito laboral. Ahora mismo, la Inspección de trabajo no tiene estas competencias de declarar nulo un despido o de sancionar represalias por denuncias, con lo que habrá que modificar su estatuto y reforzar sus plantillas. Además, existen cierto tipo de protecciones que la Inspección de trabajo difícilmente podrá garantizar o gestionar, como la ayuda psicológica, la ayuda jurídica frente a demandas de todo tipo al denunciante, o la determinación de indemnizaciones por daños o perjuicios no laborales. Todo ello requiere probablemente que exista en la Agencia externa pública una unidad especializada en protección a los denunciantes en el sector privado, unidad que tendrá que trabajar con la Inspección de trabajo de forma coordinada. En suma, parece necesaria la creación de una Agencia Estatal con competencias amplias de prevención y lucha contra la corrupción y fraude, que tendría como una labor esencial la protección a los alertadores y denunciantes, tanto del sector público como del sector privado, con una clara independencia orgánica y funcional. También es cierto que, en el ámbito autonómico, se necesitará establecer un sistema de protección a los denunciantes mediante una agencia autonómica que reúna las condiciones de independencia que, por ejemplo, tiene la Agencia Valenciana Antifraude, cuyo trabajo es del todo encomiable (Sierra, 2020b). Agencias, además, con competencias para el control de la Administración local respectiva. La agencia estatal podría asumir competencias para las Comunidades Autónomas que no desearan crear su propia agencia.

La siguiente reflexión tiene que ver con las personas que podrían denunciar y ser protegidas. Para empezar, no existe una definición legal común de lo que es whistleblower (OCDE, 2011). De acuerdo con numerosos autores, el alertador o denunciante es una persona que informa sobre hechos que se producen en torno a su propia organización y lo hace sin necesidad de desempeñar funciones específicas de control (Ragués y Vallés, 2013). La Organización Internacional del Trabajo (OIT) la define, en su Tesauro, como "la denuncia por parte de empleados o ex empleados de prácticas ilegales irregulares, peligrosas o poco éticas de los empleadores". La Convención Civil del Consejo de Europa sobre la corrupción se refiere a "los empleados que tienen motivos razonables para sospechar de la corrupción y que informan de buena fe su sospecha a personas o autoridades responsables" (1999, artículo 9). La Directiva Europea se refiere a quienes obtienen la información que comunican con motivo de sus actividades laborales y, por tanto, corren el riesgo de represalias laborales, por ejemplo, por incumplir la obligación de confidencialidad o de lealtad. Aunque también ampara a proveedores, consultores, contratistas y subcontratistas, voluntarios y trabajadores en prácticas, facilitadores, compañeros de trabajo o familiares del denunciante e, incluso, a la entidad jurídica de la que el denunciante sea propietario, para la que trabaje o con la que esté relacionado en un contexto laboral. Más aún, también protege a los responsables de la gestión de las denuncias y su investigación interna. Finalmente, y sin ánimo exhaustivo, la Convención de Naciones Unidas contra la corrupción, en su artículo 33, los define como "toda persona que denuncie de buena fe y por motivos razonables a las autoridades competentes cualquier hecho relativo a delitos tipificados con arreglo a la presente Convención". De todo este conjunto de definiciones surgen al menos tres temas de debate. El primero es si sólo debe protegerse a quienes conocen de los hechos en el marco de una relación de 
trabajo o si se incluye también a cualquier persona que denuncia. El segundo, trata de si se incluye como objeto de la denuncia sólo actuaciones vinculadas a corrupción o todo tipo de irregularidades o conductas no éticas. El tercero, si para ser considerado alertador es preciso actuar o no de buena fe. El modelo autonómico español tiende a aplicar de manera extensiva el estatuto del denunciante a los ciudadanos, en todo lo que sea posible y esté dentro de sus atribuciones - incluso si se trata de personas jurídicas-, especialmente en el modelo valenciano (Sierra, 2020b: 22). Ciertamente, esta opción no es la de la Directiva europea, que sólo protege en el marco de un contexto laboral. Esto puede ser positivo, pero hace mucho más compleja la gestión del sistema. En relación al segundo debate, no está claro en la directiva si las denuncias por incumplimientos éticos están incluidas, aunque parece que no, pues sólo habla de denuncias en fraudes e incumplimientos normativos. No obstante, parece que los incumplimientos de códigos éticos deberían incluirse cuando tuvieran una dimensión que afecte suficientemente al interés general. Con esto, de nuevo, se complejiza más la gestión. Finalmente, la Directiva no exige la buena fe, abriendo la vía a cualquier tipo de razones para justificar la denuncia. Con todo esto, podemos asegurar que la implementación eficaz de la norma se hará aún más difícil. Y ello sin considerar la necesidad de generar algún sistema especial para las denuncias en las áreas de seguridad nacional e inteligencia.

Finalmente, la pregunta que nos queda es: ¿quién va a controlar que se cumplen las normas previstas en la ley? ¿habrá sanciones por incumplimiento? ¿quién sanciona en el sector público y en el sector privado? En Francia, la Agencia Francesa Anticorrupción es la encargada no sólo del control, sino también de las sanciones y de ir generando recomendaciones a las organizaciones para que prevengan adecuadamente conductas no éticas en las organizaciones. En España, la Agencia central anticorrupción debería asumir esa labor también.

\section{TEORÍA CAUSAL Y REgLAS DE DECISIÓN. EL PROBLEMA DE LA GESTIÓN INTERNA DE LAS DENUNCIAS. ¿POR QUÉ LA GESTIÓN INTERNA ES A MENUDO INSUFICIENTE?}

La Directiva europea incentiva la gestión interna de las denuncias, sin por ello dejar de abrir la puerta a denuncias externas cuando la vía interna falla o es previsible que falle. La razón fundamental es claramente utilitarista, se trata de evitar escándalos que generan el descrédito de empresas y, con ello, la puesta en peligro de su supervivencia, o, en el caso de lo público, la deslegitimación de las instituciones. Es decir, por una parte, se incentiva la denuncia, a efectos de evitar que el incumplimiento de las normas se generalice y, con ello, la competencia justa se destruya y el Estado de derecho se diluya. Y, por otra, se pretende que las investigaciones y las decisiones para resolver el conflicto se realicen internamente, "lavando los trapos sucios en casa". El mejor de los mundos es, en consecuencia, aquel en el que hay denuncias internas en caso de cualquier incumplimiento normativo que dañe el interés general y, al tiempo, aquel en el que todo eso se resuelve sin escándalo y sin salir de casa. A nuestro juicio, esta opción tiene varias debilidades.

Empecemos con el sector privado. La primera es que no resuelve bien las paradojas internas que genera, incluso en organizaciones no estructuralmente corruptas (Cailleba y Charreire, 2018). Las principales corrientes de la economía se basan en una idea de la naturaleza humana conocida sintéticamente como la del "homo economicus". Según esta 
teoría, los seres humanos buscan maximizar su propio interés, un interés que podría reducirse a la satisfacción obtenida por la adquisición y el consumo de bienes. Como señala Adam Smith en La riqueza de las naciones, un sistema económico de mercado se basa en el concepto de que cada agente económico persigue sus propios intereses y, persiguiéndolos inteligentemente, construye un sistema productivo eficiente (Smith, 1937: 14). Este modelo de ser humano, si no se controla con un observador imparcial que, desde dentro de la conciencia, guíe racionalmente, puede cometer numerosos fallos morales. Para empezar, ante el dilema de respetar las reglas abstractas del mercado justo o promover los intereses de la empresa, sobre todo en momentos de crisis, buscará satisfacer sus propias preferencias y, a menos que vea claramente que el incumplimiento de las normas pone en riesgo su libertad o a su empresa, podrá llegar a pagar sobornos o fijará precios con la competencia para subsistir. La empresa es una "organización social estructurada y coordinada en su actuación, con la función de contribuir, con o sin ánimo de lucro, a la satisfacción de las necesidades humanas mediante la generación de bienes y servicios" (Cabanes, 2017: 192), pero ello sólo lo puede hacer si es rentable o se sostiene económicamente. La decimocuarta edición de la Encuesta Mundial sobre el Fraude de Ernst \& Young, realizada en 2015, ofrece información de 2.825 altos ejecutivos de 62 países de todo el mundo. Según la encuesta, más de un tercio consideraba que el soborno y la corrupción se daban de forma generalizada en su país, y casi la mitad podía justificar un comportamiento poco ético para alcanzar objetivos financieros (Ernst \& Young, 2016). Estas cifras ilustran claramente las tensiones que existen en las empresas entre las posibles desviaciones y su divulgación a través de las denuncias. Con todo esto en mente, cualquier persona que quiera alertar tiene un debate interno entre no denunciar actuaciones que pueden ser buenas competitivamente para su empresa (no quebrar la lealtad), y actuar de acuerdo con los principios morales que le llevan a rechazar la ilegalidad y la injusticia. Por su parte, la empresa se enfrenta a otra paradoja, esta vez organizacional, dado que tiene que favorecer todo un sistema de denuncias, incluso anónimas, que pueden poner en riesgo la supervivencia de la propia empresa. Finalmente, existe una paradoja organizacional, en la que la estrategia de generar empleados y directivos creativos, innovadores, que asumen riesgos, se enfrenta a la posibilidad de denuncias internas de todo tipo que llevarían al conservadurismo y a la inamovilidad.

Para que este tipo de tensiones y paradojas no genere respuestas ilícitas e inmorales, sería preciso un proceso de desarrollo moral entre los empresarios y directivos que facilitara la toma de decisiones guiada por principios universalizables y no por intereses cortoplacistas. Las empresas es cierto que tienen que ser rentables o, al menos, sostenibles, pues sin ello no podrán cumplir su misión, pero guiarse por la búsqueda de rentabilidad no basta para que el sistema de mercado funcione. Si el mercado ignora a la sociedad en la que opera y rompe sus propias reglas del juego -esencialmente la competencia justa- tendrá un impacto negativo en su propia actividad y, en última instancia, atentará contra su propia supervivencia, como acabamos de ver en la Gran Depresión; finalmente, cuando llegue la crisis, será necesaria la intervención pública para salvar al propio mercado de sus excesos (Polanyi, 2002), eso sí, con dinero de todos. Para evitar estas tensiones destructivas, el mercado debe entenderse como parte del sistema social y, por tanto, como un socio clave en el desarrollo del valor social y de las políticas económicas que sirven esencialmente al bien común. El valor social implica productos y resultados no financieros, es decir, intervenciones que implican a las personas, las comunidades, el capital social y el medio ambiente y que mejoran el bienestar social (Porter y Kramer. 2011; Mulgan, 2010). Por 
eso es importante incluir una dimensión moral en la toma de decisiones en este ámbito, más compleja que el utilitarismo mal entendido o utilitarismo ad hoc (Sandin, 2009). Desde un punto de vista económico, y respetando la noción de que el hombre persigue su propia felicidad y huye del dolor, sería necesario, a través de la educación, introducir el utilitarismo de la regla en el sistema para las decisiones empresariales de esta naturaleza, ya que: "Permite que un acto particular en una ocasión particular sea adjudicado como correcto o incorrecto según si está de acuerdo con una regla útil o la viola; y una regla se juzga útil o no por las consecuencias de su práctica general" (Duignan y West, 2017). Esto implicaría que los actores económicos -como principio de conducta en este tipo de dilemabuscarán el mayor bien para el mayor número de personas sin hacer un balance de los beneficiados o perjudicados por cada acción, si no, más bien, siguiendo las reglas que la historia y la calidad de los resultados han demostrado que producen el mayor bien para el mayor número de personas (Mill, 1863: 35-37). Como quiera que la competencia justa es una regla clave para el buen funcionamiento del mercado, promover y asumir este modelo de reflexión moral parece esencial para el adecuado funcionamiento de un buen modelo de ética en la empresa. Esto significa que hay que asumir que el respeto a las reglas de la competencia leal (tal y como la entienden la mayoría de los estudiosos de la ética empresarial en la actualidad) conduce a un mayor bien social que romper dichas reglas y generalizar el juego sucio. En conclusión, a menos que las reglas entren en conflicto entre ellas, es mejor seguirlas, sin siquiera calcular las consecuencias, que sopesar el respeto a las reglas con el beneficio inmediato para la empresa. Mientras este nivel de desarrollo moral no sea generalizado en el mundo empresarial, y se manifieste en una cultura organizativa donde el liderazgo ético y la responsabilidad social estén profundamente insertos, es muy difícil que la mera gestión interna de denuncias pueda producir resultados positivos (Treviño et al., 1999).

Situándonos en el ámbito de lo público, el problema de la gestión interna de denuncias no tiene que ver con la lucha por la rentabilidad y subsistencia de la organización, sino con la segunda debilidad del modelo: la de que no considera los factores de conformidad, socialización y corrupción organizada que se dan en bastantes organizaciones públicas y privadas. Pasaremos a analizar ahora este problema. Cuando una persona se une a una organización, entra en un entorno que no solo produce incentivos y desincentivos, sino que también añade significado. Por ello, las personas tienden a seguir las reglas de juego internas de su organización (De Graff et al. 2010; Arellano, 2017). En general, las reglas normativas pueden variar drásticamente de una empresa a otra, por lo que no puede haber una conducta específica que sea, en sí misma, desviada. Más bien, esta desviación depende de la violación de las normas de algún sistema organizativo. El problema es que la conformidad no garantiza la moralidad, ya que, en ocasiones, las normas y órdenes internas son inmorales, y seguirlas conduce a la corrupción (Etzioni, 2017). En definitiva, se puede ser a la vez un buen trabajador y un delincuente. Cuando se desarrollan prácticas corruptas dentro de una organización y no hay consecuencias adversas para los corruptos, se produce un fenómeno de normalización que puede tener consecuencias devastadoras (Ashforth y Anand, 2003). En otra encuesta realizada en 2007 (PricewaterhouseCoopers, 2007), en más de 5.400 empresas de 40 países, casi un tercio informó de robos y malversaciones internas. Una organización puede pasar de ser ética, a tener casos aislados de corrupción y, posteriormente, debido a la impunidad, la organización se convertiría en una de individuos corruptos $\mathrm{y}$, en última instancia, en una organización corrupta en sí 
misma si no se detienen los procesos por los que se normaliza la corrupción (Pinto et al., 2018).

Esta normalización se deriva de tres procesos que se refuerzan mutuamente (Ashforth y Anand, 2003). El primero es a través de la institucionalización, donde una decisión corrupta se toma en un momento dado y permanece dentro de las estructuras y los procesos, por lo que se convierte en rutina. La segunda es a través de la racionalización y la justificación, donde se desarrollan historias e ideas a favor de los individuos corruptos para justificar e incluso valorar la corrupción (Zyglidopoulos y Fleming, 2008). Estas justificaciones pueden ir desde mantener que (1) la acción no causó ningún daño a nadie o que no es técnicamente ilegal; hasta (2) "todo el mundo lo hace" y eso es lo que mis colegas esperan de mí; o hasta (3) que es necesario para la supervivencia de la organización (Aguilera y Vadera, 2008). La tercera es a través de la socialización, por la que se lleva a los neófitos de una organización a ver la corrupción como algo normal e incluso deseable (Milgram, 2005). Estos procesos de normalización deberían servirnos de advertencia, una vez más, de la tendencia a asumir que defender de forma poco ética los intereses de una organización en un momento determinado (y aislado) no es corrupción y, por tanto, no hay que aplicar sanciones. Quien emprende el camino del fraude y la corrupción tendrá cada vez más dificultades para cambiar de rumbo a medida que vayan surgiendo fenómenos incrementales difíciles de controlar. La experiencia práctica nos demuestra que, una vez que se toma este camino, es muy difícil salir de él, y la expansión de los actos inmorales destruye la legalidad, lo que tiene consecuencias nefastas. Denunciar la corrupción o ilegalidad en entornos con fuerte socialización de equipo, cuando están arraigadas prácticas inmorales, es muy difícil para cualquier persona (Butcher, 2020).

Por último, cuando hablamos de organizaciones corruptas, normalmente nos encontramos con una cúpula directiva que es plenamente consciente de que está utilizando estrategias y acciones corruptas para su beneficio personal y, en ocasiones, el de la empresa. Esto podría clasificarse como corrupción organizativa: un delito que se comete utilizando la autoridad dentro de las organizaciones para obtener un beneficio personal (Aguilera y Vadera 2008: 433). Por ello, (véase Pinto et al., 2010: 694), los procesos de normalización se diseñan estratégicamente para crear una organización que favorezca esta dinámica, muchas veces ocultando y separando claramente los roles y tratando de crear un cortafuegos que reduzca el riesgo legal para la dirección y jugando con la identidad corporativa como factor de socialización. En cualquier caso, los factores exógenos suelen ser claves a la hora de explicar cómo se extiende este fenómeno (Pinto et al., 2010: 69699), sobre todo en lo que se refiere a la competencia feroz y la escasez de recursos en el ámbito empresarial, la estructura y el sistema de adjudicaciones de una misma industria (por ejemplo, las que están muy reguladas, véase Szwajkowski, 1985) o la cultura de cohesión del sector económico correspondiente cuando admite o fomenta prácticas colusorias o corruptas.

De acuerdo con nuestros propios datos empíricos (Ver Palomo et al., 2018) los sobornos entre empresas son altos en España. El 17,6\% de las/os empresarios que encuestamos reconocía que los interlocutores de otras empresas se mostraban a menudo dispuestos a ofrecer algo a cambio de un trato preferente. Para entender las dimensiones del fenómeno, es interesante ver qué porcentaje de personas reconoce haber sido requerido para pagar un 
soborno dentro del sector público español. Según los eurobarómetros (CE, 2017-2020), sólo el 2 o $3 \%$ admite haber sido requerido a pagar un soborno en los últimos 12 meses en España, es decir, el soborno público parece bajo en España. Este hallazgo inesperado (el soborno en España, y probablemente en otros países, está más extendido en el sector privado que en el público) es, sin duda, una llamada de atención para entender el problema de la denuncia e investigación interna en las empresas. Ahora bien, este hecho no nos debe llevar a pensar que las organizaciones públicas están libres de los problemas de corruptelas, ilegalidades, fraudes y despilfarro y que, por ello, las denuncias internas serán investigadas y resueltas con toda rapidez y sin represalias para los denunciantes. Para empezar, los sobornos en España son bajos entre los empleados públicos, pero no tan bajos entre la cúpula política. Los grandes casos de corrupción han afectado a las cúpulas de las organizaciones públicas y, aunque se establezcan protecciones a los futuros denunciantes, las posibilidades de que se incumplan los deberes de confidencialidad y no represalia cuando se denuncian casos que afectan a la estructura de mando de la organización (alcaldes, consejeros, presidentes y vicepresidentes autonómicos, ministros...) es alta. Pero la mayoría de las denuncias que se verán, si se abre esta posibilidad, tendrán que ver con clientelismo y privilegios concedidos a miembros de las redes políticas correspondientes, con fraudes más o menos consentidos, con despilfarros guiados por el interés en ganar las elecciones próximas. En todos esos supuestos, es la dirección de la organización la que está implicada y, por ello, la destrucción de pruebas, la investigación sesgada y la represalia estarán garantizadas.

Con todo esto en mente, parece muy difícil que las investigaciones internas lleguen a buen puerto en numerosos casos y que los denunciantes, en el sector público y privado, no sufran represalias. En general, incentivar que las personas que denuncien pasen por esta fase interna, en España, puede ser inconveniente, aun cuando la clave es aportar una información clara de los caminos a seguir, de las reglas existentes y de los riesgos y posibilidades, para que cada persona opte, sabiendo bien las posibles consecuencias (OCDE, 2016). De ahí la importancia de la información y formación en estos derechos, así como de generar un sistema de denuncia externa y un sistema externo de investigación y protección independiente (Amoedo, 2017).

\section{VARIABLES NO NORMATIVAS}

Al final, para que la implementación se pueda realizar con unas mínimas garantías de éxito (el éxito total es imposible), se requiere también que la sociedad, los medios de comunicación, la clase política, el entorno económico y tecnológico ayuden. En el caso de la trasposición de la Directiva (UE) 2019/1937 nos encontramos con una situación económica ciertamente difícil como consecuencia de la pandemia generada por la COVID 19. Va a ser necesario que se considere como una estrategia de resiliencia la consolidación de instituciones de buena gobernanza en España, y esto depende claramente de los políticos en el gobierno y, también, en la oposición. Sin el apoyo político y su implicación será imposible que este conjunto de normas y decisiones llegue a buen puerto. Un elemento que se deriva de ello es la calidad de las personas que se pongan al frente de las organizaciones responsables de implementar la ley y sus reglamentos; si se optara por personas competentes, independientes de intereses partidistas y fuertemente comprometidas con la calidad democrática, entonces habría al menos un motor impulsor con suficiente fuerza y energía. Se necesita de ese motor moral, como se necesita de unos 
presupuestos suficientes para poder llevar adelante todo el conjunto de medidas que se derivan de esta mejora institucional.

Más optimismo genera el cambio tecnológico. La tecnología está transformando la denuncia de irregularidades al potenciar la denuncia anónima y cambiar la naturaleza de la propia alerta. Por ejemplo, los portales web están sustituyendo a los servicios tradicionales de líneas directas, con las ventajas adicionales del anonimato, al tiempo que facilitan la aportación de documentación de apoyo y la comunicación con una autoridad investigadora sin problemas de filtraciones. Las aplicaciones para teléfonos móviles también incluyen ya la posibilidad de hacer denuncias de forma anónima y adjuntar fotos o documentos. Blockchain, por su parte, es uno de los desarrollos tecnológicos más prometedores para los denunciantes, aunque aún no se ha hecho plenamente realidad. WhistleAI, Crypto Community Watch y Darkleaks son algunas de las pocas plataformas de denuncia basadas en blockchain que existen actualmente. Las tecnologías de cadena de bloques permiten alcanzar el equilibrio entre el deseo de anonimato de los denunciantes y la necesidad de comunicación continua de las autoridades investigadoras. Los datos cargados en una plataforma basada en la cadena de bloques son inmutables, lo que significa que los empleadores u organizaciones implicados en la revelación no pueden borrar o manipular las pruebas que se han agregado en bloques interconectados (Feinstein y Devine, 2021: $68)$.

Uno de los temas que siempre ha generado más resquemor a apoyar la denuncia ha sido el del rechazo cultural al "chivato" (McClain y Seifert, 2018; Pérez Monguió, 2019). Sobre todo en países que viven o han vivido dictaduras o que aún tienen vagos recuerdos de los procesos inquisitoriales, este rechazo cultural existe. No obstante, el cambio cultural en este ámbito es enorme e imparable. Por ejemplo, en España, de acuerdo a una reciente encuesta impulsada por la ONG Blueprint For Free Speech (2021) en colaboración con la consultora internacional IPSOS, siete de cada diez españoles/as piden proteger a los alertadores de corrupción aunque revelen informaciones secretas. En esta encuesta - con 2.174 entrevistas realizadas a nivel nacional- se puede comprobar que las mujeres y las personas con formación más elevada son las que más apoyan a los alertadores (en un $73 \%$ ), incluso si revelan información reservada, por encima del conjunto del $71 \%$ de todos los encuestados. No obstante, incluso en el segmento de resultado más bajo (el de los que sólo han recibido educación primaria) ese apoyo es superior a los dos tercios (67\%). Por profesión o empleo, el apoyo más elevado a los alertadores se registra entre los ejecutivos y profesionales (73\%), pero es casi tan alto (72\%) entre los técnicos, administrativos y funcionarios. Una vez más, el menor apoyo (el que se da en las respuestas de obreros, gente con empleos básicos y miembros de las fuerzas armadas) es superior a los dos tercios $(67 \%)$.

Finalmente, una variable clave para el éxito implementador es el del apoyo de los medios y de la sociedad civil (Cerrillo, 2018). El ejemplo en los países anglosajones es muy claro al respecto. En el Reino Unido, las campañas de sensibilización promovidas por diversas asociaciones fueron claves para que el tema entrara en la agenda gubernamental. Entre las fundaciones que más han promovido el whistleblowing, la Public Concern at Work es la más conocida (Vestri, 2019). En Estados Unidos el papel del Government Accountability Project ha sido esencial para mantener una agenda de mejoras en la legislación y de preocupación social por el problema. A nivel internacional, XNet, 
Blueprint For Free Speech o Transparency International con sus estudios (2009) han sido claves en la generación de la Directiva europea y lo son en la presión por su trasposición.

\section{CONCLUSIONES}

La trasposición de la Directiva europea, aunque recoja en su articulado las mejores prácticas internacionales, supone un reto de implementación enorme. Este texto ha tratado de explicar una gran parte de las demandas organizativas, financieras, de gobernanza que genera. Con ello, se recupera el foco en la implementación de políticas como un área que merece la atención de las policy sciences. En este caso, basado en experiencia empírica internacional, se demuestra que, a pesar de las normas, para alcanzar un mínimo éxito en la protección al alertador, es preciso considerar variables como la complejidad del tema, la multiplicidad de actores, la amplitud de las posibles demandas, la dualidad de sistemas internos y externos, públicos y privados, y la heterogeneidad de espacios de protección y sanción.

Este texto tiene limitaciones, dado que la política de protección está aún por nacer y tan solo existe una Directiva a trasponer, ni siquiera un proyecto de ley detallado. El caso está naciendo y su análisis completo tendrá que llegar cuando las normas y decisiones se adopten. Pero ello puede hacer aún más útil estas reflexiones si, con ellas, los redactores del proyecto ya anticipan problemas y les dan respuestas normativas coherentes. Aún así, de lo analizado surgen posibles líneas de investigación de gran calado. Una de ellas es la de los límites y restricciones de las estrategias de isomorfismo coercitivo y normativo en las políticas de buena gobernanza (Powell y DiMaggio, 1999); otra tiene que ver con el estudio en este caso del peso de la dependencia, los equilibrios puntuados, la importancia de las ideas y las coyunturas críticas, y la tensión entre las fuerzas políticas en juego y sus puntos de veto (Baumgartner y Jones, 1993; Tsebelis, 2000). Finalmente, entrando en el análisis del "espacio de acción" (Ostrom, 2005), será relevante el estudio del papel de las asimetrías de poder y su influencia en el cambio.

\section{BIBLIOGRAFÍA}

Aguilera, R. V. and Vadera, A. K. (2008) The Dark Side of Authority: Antecedents, Mechanisms, and Outcomes of Organizational Corruption. Journal of Business Ethics 77: 431-449.

Alford C. F (2002) Whistleblowers: Broken Lives and Organization. Fred. Ithaca, New York: Cornell University Press.

Amoedo Barreiro, D. (2017). Elementos esenciales para un sistema de protección de denunciantes. Revista Internacional de Transparencia e Integridad, 4, pp. 1-8.

Arellano, D. (2017) "Corruption as an organizational process: Understanding

the logic of the denormalization of corruption." Contaduría y Administración 62 (2017) 827-842.

Ashforth, B. E., \& Anand, V. (2003) The normalization of corruption in organizations. Research in Organizational Behavior, 25: 1-52.

Baumgartner, F.R y Jones, B.D. 1993. Agendas and Instability in American Politics. Chicago: Chicago University Press

Benítez Palma, E. (2018). El control externo y el whistleblowing (canales de denuncia). Revista Española de Control Externo, 59, pp. 11-42.

Blueprint For Free Speech (2021). Encuesta sobre persecución judicial de los denunciantes de prácticas corruptas. https://m.facebook.com/Blueprintforfreespeech/posts/?ref=page_internal\&mt_nav=0 
Brown, A. J., Mazurski, E. y Olsen, J. (2008). The incidence and significance of whistleblowing. En A. J. Brown, Whistleblowing in the Australian Public Sector Book

Australia: ANU Press.

Butcher, D. 2020. Lessons from an MLB pitcher and whistleblower. The case of baseball pitcher Mike Fiers, who spoke out about the Houston Astros' sign stealing, illustrates the difficulty of being a whistleblower in a team setting. Strategic Finance (November): 38-45.

Cabanes, Manuel (2016) La misión de la empresa como objetivo supremo de la misma: análisis discursivo y mirada prospectiva. 189-216 Icade. Revista cuatrimestral de las Facultades de Derecho y Ciencias Económicas y Empresariales, $\mathrm{n}^{\circ}$ _99 septiembre-diciembre 2016: 189-216

Cailleba, P. y Charreire Petit, S. (2018) The whistleblower as the personification of a moral and managerial paradox.M@n@gement 2018/1 (Vol. 21): 675-690.

Capdeferro Villagrasa, O. (2020). El paper de 1'Oficina Antifrau de Catalunya en la lluita contra la corrupció en el sector públic català. Anàlisi i propostes de reforma amb motiu del seu $10 \mathrm{E}$ Aniversari. Revista de Dret Public, 60, pp. 35-64. doi: https://doi.org/10.2436/rcdp.i60.2020.3413

Cerrillo i Martínez, A. (2018). Diez propuestas para la colaboración ciudadana en la alerta de malas prácticas en la Administración Pública. Revista Internacional de Transparencia, 6, pp. 1-7.

Dror, Y. (1983) Public Policy Making Reexamined. New York: Transaction Publishers

De Graaf, G., Wagenaar, P., \& Hoenderboom, M. (2010). Constructing corruption. In G. de Graaf, P. von Maravic, \& P. Wagener (Eds.), The good cause. Theoretical perspectives on corruption (pp. 98-114). Opladen \& Famington Hills: Barbara Budrich Pub.

Diugnan, B and H. R. West (2017) "Utilitarianism." Encyclopadia Britannica. Encyclopædia Britannica, inc., date Published: November 15, 2017, URL:

https://www.britannica.com/topic/utilitarianism-philosophy. Access Date: September 7, 2018.

Dworkin,. T. M. (2007). SOX and Whistleblowing. Michigan Law Review

Volume 105 Issue 8: 1757-1780.

Ernst \& Young (2016). Enquête internationale sur la fraude 2016. 14ème édition. Consultada el 28 enero 2021. http://www.ey.com/FR/fr/Newsroom/News-releases/ey-communique-de-presseenquete-internationale-ey-sur-la-fraude-2016

Etzioni, A. (2017) The Moral Wrestler: Ignored by Maslow. Symposium: Revisiting Maslow: Human Needs in the 21st Century. Springer: Published online on 10/18/2017. Retrieved December 1, 2017. https://doi.org/10.1007/s12115-017-0200-3.

Feinstein, S. y Devine, T. (2021) Are whistleblowing laws working? A global study of whistleblower protection litigation. Washington D.C. y Londres: Government Accountability Project and the International Bar Association.

Glazer, M.P and Glazer, P.M. 1991. Whistleblowers. Exposing Corruption in Government and Industry. Basic Books. New York

Garrido Juncal, A. (2019). La protección del denunciante: regulación autonómica actual, novedades normativas y propuestas de futuro. REALA, 12 (nueva época), pp. 126-151. doi: https://doi.org/10.24965/reala.i12.10731

Hill, M. y Hupe, P. (2002) Implementing Public Policy: Governance in Theory and in Practice. London: Sage

Lindblom, C.E. (1965) The intelligence of Democracy: Decision Making through Mutual Adjustment. New York: Free Press

McClain, G. and D. Seifert. 2018. The impact of moral identity and societal culture on whistleblowing: A comparison between the United States and France. Journal of Forensic \& Investigative Accounting 10(3): 357-373.

Milgram, S. (2005) Obedience to authority. New York: Printer \& Martin.

Mill, John Stuart (1863) Utilitarianism (1 ed.). London: Parker, Son \& Bourn, West Strand. Retrieved April 6, 2018 via Google Books 43.

Mulgan, G. (2010) “Measuring Social Value.” Stanford Social Innovation Review, (Summer): 38- 
OCDE (2011) Study on Whistleblower Protection Frameworks: Compendium of Best Practices and Guiding Principles for Legislation. Informe para el G20 Anticorruption Working Group, Protection of Whistleblowers París: OCDE

OECD (2016) Committing to Effective Whistleblower Protection. París: OCDE

Ostrom, Elinor. (2005) Understanding Institutional Diversity. Princeton: Princeton University Press

Palomo, J., Villoria, M., García, G., Perianes, A. (2018) Private Corruption:

research results in Spain. En De Nicola (ed.) The Private Corruption Barometer. Drafting and piloting a model for a comparative business victimization survey on private corruption in the EU, Università degli Studi di Trento.. pags. 81-95.

Pinto, J., Leana, C.E., Pil, F.K. (2008) "Corrupt organizations or organizations of corrupt individuals? Two types of organization-level corruption." Academy of Management Review, Vol. 33, No. 3, 685-709.

Pérez Monguió, J. M. (2019) "Del chivato al cooperador: el whistleblowing”, RVAP, núm.115, 2019: 345-375.

Ponce Solé, J. (2017a). Las agencias anticorrupción. Una propuesta de lista de comprobación de la calidad de su diseño normativo. Revista Internacional de Transparencia e Integridad, 3, pp. 1-13.

Ponce Solé, J. (2017b). El derecho a una buena administración y el derecho administrativo iberoamericano del siglo XXI. Buen gobierno y derecho a una buena administración contra arbitrariedad y corrupción. En Alonso Regueira, E. (dir.) El control de la actividad estatal: 219-247. Buenos Aires: Facultad de Derecho-UBA

Polanyi, K. (2002) The Great Transformation: The Political and Economic Origins of Our Time. Boston: Beacon Press.

Porter, M. \& Kramer, M. (2011) Creating Shared Value. Harvard Business Review, 89, pp. 62-77.

Powell, W. y Di Maggio, P. (1999). El Nuevo Institucionalismo en el Análisis Organizacional. México: Fondo de Cultura Económica

Pressman, j y Wildavsky, A. (1984) Implementation: How Great Expectations in Washington Are Dashed in Oakland. Berkeley: University of California Press.

PricewaterhouseCoopers (2007), Economic Crime: People, Culture and Controls: The 4th Biennial Global Economic Crime Survey. London: PricewaterhouseCoopers.

Raguès Vallès, R., (2013) Whistleblowing. Una aproximación desde el Derecho Penal, Madrid: Marcial Pons.

Romero, J., Brandis, D., Delgado Viñas, C., García Rodríguez, J. L., Gómez Moreno, M. L., Olcina, J., Rullán, O., Vera-Rebollo, J. F., \& Vicente Rufí, J. (2018). Aproximáción a la Geografía del despilfarro en España: balance de las últimas dos décadas. Boletín de la Asociación de Geógrafos Españoles, 77, 1-51.

Sabatier, P. y D. Mazmanian (1980) The implementation of public policy: a framework of analysis . Policy Studies Journal, Volume 8, Issue 4, January 1980: 538-560

Sandin, Per (2009) Approaches to Ethics for Corporate Crisis Management. Journal of Business Ethics volume 87, 109 (2009) https://doi.org/10.1007/s10551-008-9873-2.

Sierra Rodríguez, J. (2020a). Impulso europeo al whistleblowing y las autoridades de integridad. Eunomía. Revista en Cultura de la Legalidad, 19, pp. 64-85.

Sierra Rodríguez, J. (2020b) Anonimato y apertura de los canales de denuncia de la corrupción. Revista General de Derecho Administrativo 55 (Iustel)

Smith, Adam (1937) The Wealth of Nations, New York: Random House, 1937.

Stanger, A. (2019a). Whistleblowers: Honesty in America from Washington to Trump. New Haven. Yale University Press.

Stanger, A. (2019b). The Personal Cost of Whistleblowing. New America Weekly. Oct. 10, 2019

Szwajkowski, E. (1985) Organizational illegality: Theoretical integration and illustrative application. Academy of Management Review, 10: 558-567.

Transparency International (2009), Alternative to Silence: Whistleblower Protection in 10 European Countries, Transparency International, Berlin. 
Trevino, L., Weaver, G. R, Gibson, D., \& Toffler, B. (1999). Managing ethics and legal compliance: What works and what hurts. California Management Review, 41(2), 131-150.

Tsebelis, George (2000) Veto Players and Institutional Analysis. Governance, Vol. 13, No. 4, October: 441-474)

Vestri, G. (2019) Aproximación al sistema de «whistleblowing». Un nuevo

desafío para la Administración Pública española. Revista General de Derecho Administrativo (Iustel, mayo 2019), 51.

Zyglidopoulos, S., \& Fleming, P. (2008) Ethical distance in corrupt firms: How do innocent bystanders become guilty perpetrators? Journal of Business Ethics, 78(172.), 4-5. http://dx.doi.org/10.1007/s10551-007-9378-4

\section{Breve Curriculo:}

Manuel Villoria

Catedrático de Ciencia Política (Universidad Rey Juan Carlos-Madrid), dirige el Observatorio en Buena Gobernanza-URJC. Tiene 5 sexenios de investigación/transferencia y 3 tramos de docentia acreditados. Es Director del Máster de Alta Dirección Pública del Instituto Universitario Ortega y Gasset (IUOG)-UIMP y del Máster en Gestión de la Seguridad, crisis y emergencias IUOG-URJC; Doctor en Ciencia Política y Sociología por la Universidad Complutense de Madrid, Licenciado en Derecho y Licenciado en Filología; fue becario Fulbright en USA, donde realizó cursos de Master y Doctorado por la Indiana University. Co-fundador y miembro de la Junta Directiva de Transparency International, capítulo español. 